\title{
Persistent low back pain reveals a massive non-ruptured aortic abdominal aneurysm: A case report
}

\author{
Jorge Vaz Lourenço, Ricardo Marques, Daniela Marado, \\ Adriano Rodrigues
}

\begin{abstract}
A 62-year-old man presented with an insidious low back pain that radiated to both knees. A pulsatile abdominal mass was detected on physical examination. Imaging study identified a pararenal aortic abdominal aneurysm measuring $20 \mathrm{~cm}$ long and with a caliber of 10 $\mathrm{cm}$. The aneurysm was surgically repaired. The patient survived the procedure and has shown a positive recovery.
\end{abstract}

Keywords: Aortic aneurysm, Endovascular repair, Low back pain

\section{How to cite this article}

Lourenço JV, Marques R, Marado D, Rodrigues A. Persistent low back pain reveals a massive nonruptured aortic abdominal aneurysm: A case report. Case Rep Int 2018;7:100050Zo6JL2018.

Article ID: 100050Zo6JL2018

$* * * * * * * * *$

doi: 10.5348/100050Zo6JL2018CR

Jorge Vaz Lourenço', Ricardo Marques', Daniela Marado, Adriano Rodrigues ${ }^{1}$

Affiliation: ${ }^{1}$ Serviço de Medicina Interna, Centro Hospitalar e Universitário de Coimbra, Portugal.

Corresponding Author: Jorge Vaz Lourenço, MD, Travessa da Barrosa, n¹59 5A, Vila Nova de Gaia, Portugal 4400-042; Email: jorge.v.lourenco@gmail.com

Received: 15 June 2018

Accepted: 11 July 2018

Published: 07 August 2018

\section{INTRODUCTION}

Low back pain (LBP) is the fifth cause of medical consultation in the United States [1]. Despite its relation to spinal degenerative injuries, there are other less common but serious conditions that mimic this clinical presentation, namely vascular disorders such as aortic abdominal aneurysm (AAA). It is usually defined as abdominal aorta diameter greater than $3.0 \mathrm{~cm}$ and presents with low back pain in up to $50 \%$ of patients [2].

In our daily practice, it is important not only to recognize AAA-associated risk factors but also to thoroughly screen for non-musculoskeletal signs and symptoms to improve diagnostic accuracy and patients' outcome.

\section{CASE REPORT}

A 62-year-old retired man came to the emergency department complaining of steady low back pain that had been radiating through both legs to the knee for three weeks. Pain was rated 7 out of 10 on a visual analogic pain scale. He denied fever, muscular fatigue, intermittent claudication and lower-limb paresthesia. He didn't have genitourinary or gastrointestinal symptoms. He reported no known trauma that might have caused back injury. During this period, he had been seen by his general practitioner who prescribed him metamizole 575 mg bid but the pain persisted. The patient had previous history of smoking, hypertension and ischemic heart disease with two previous myocardial infarctions (1 and 7 years ago). On physical examination, he showed normal vital signs, apyrexia and normal cardiopulmonary auscultation. On neurological examination, no motor or sensitive dysfunction was observed and Lasègue's sign was absent on both lower limbs. Abdominal examination revealed a strong pulsatile mass on light palpation of the periumbilical region without local inflammatory signs or evidence of peritoneal irritation.

Blood tests showed an elevation of serum urea (92 $\mathrm{mg} / \mathrm{dl})$ and creatinine $(1.87 \mathrm{mg} / \mathrm{dl})$, but no leukocytosis nor $\mathrm{C}$ reactive protein elevation. Abdominal ultrasound 


\section{EDORiUM Journals}

www.casereportsinternational.com

was conducted and detected an AAA measuring $9.6 \mathrm{~cm}$ of diameter, with an intramural thrombus about 4.4 cm thick. Liver, biliary tract and pancreas exploration was unremarkable. Renal ultrasound was performed, considering the evidence of renal impairment, and showed a reduced size of the right kidney associated with low corticomedullary differentiation. Given the diagnosis of symptomatic AAA, the patient was submitted to a CT angiography scan which showed a pararenal AAA with a maximum diameter of $10 \mathrm{~cm}$ and $20 \mathrm{~cm}$ long, presenting a circumferential intramural thrombus about $5.7 \mathrm{~cm}$ thick causing right renal artery stenosis. There was good vascular permeability of celiac trunk, superior mesenteric artery and left renal artery (Figure 1 ).

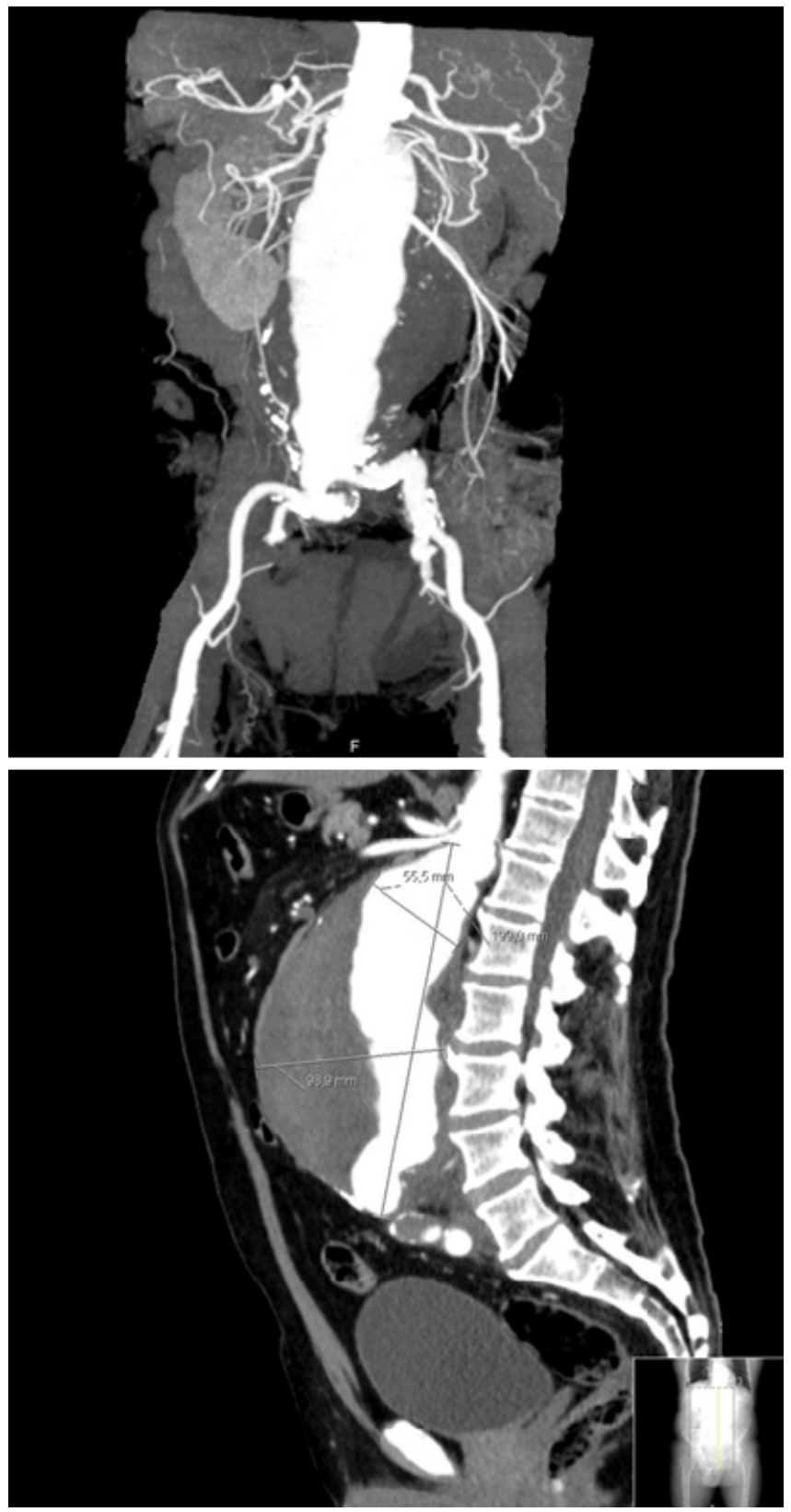

Figure 1: CT imaging showing a pararenal aortic abdominal aneurysm with a maximum diameter of $10 \mathrm{~cm}$ and $20 \mathrm{~cm}$ long, presenting a circumferential intramural thrombus about $5.7 \mathrm{~cm}$ thick and causing right renal artery stenosis.
After completing imagiological study, the patient was seen by the vascular team, who decided to perform endovascular repair of the aneurysm, a decision that was based on patient's choice and justified by the anatomic features of the AAA. The patient survived the surgery and was discharged after three weeks. He presented no postoperative vascular complications. During this period, he underwent lifestyle modifications and control of his cardiovascular risk factors, such as hypertension. After one year of follow-up, the patient had no more episodes of low back pain.

\section{DISCUSSION}

In the United States, population-based studies in adults older than 50 years have found that the prevalence of $\mathrm{AAA}$ is $3.9 \%$ to $7.2 \%$ in men and $1.0 \%$ to $1.3 \%$ in women, with an overall incidence of 7 per 1000 in those in their mid-6os. The US Preventive Services Task Force (USPSTF) published updated guidelines on the matter in 2014, concluding that there is moderate net benefit of one-time ultrasound screening for AAA in men 65 to 75 years of age with a history of smoking [3].

The Society of Vascular Surgery also recommends a one-time screening for AAA, but extends it to all men older than 65 years or as early as 55 years in men and women with a family history of AAA. Portugal estimated prevalence of AAA stands between $2-3 \%$ in screening pilot studies [4]. There is still an active debate regarding a viability of a Portuguese National AAA Screening Program [5].

An aortic dilation is considered aneurysmal if there is an increase in diameter over 50\% than expected for the same aortic segment in unaffected individuals of the same age and sex. AAAs are usually categorized in terms of etiology, morphology, size and location. This vascular disorder occurs in 3 to $9 \%$ of men aged $>50$ years and up to $28 \%$ of first-degree relatives of patients with AAAs may be similarly affected [6].

The infrarenal aorta is involved in $>80 \%$ of cases, but up to $10 \%$ of AAAs could also affect the pararenal or visceral aorta. The pathogenesis of AAAs is based on a multifactorial environment, based on the interaction between atherosclerotic disease and genetic, hemodynamic or even immunologic factors. Smoking is the only modifiable factor associated with AAA expansion, particularly if there is a minimum consumption of more than 100 cigarettes in a lifetime [7].

Other AAA-related risk factors include age (most patients are 65 years or older), sex (5 to 10 times more common in men than in women) and previous history of stroke, coronary arterial disease (with/without previous history of myocardial infarction), hypertension and hyperlipidaemia $[8,9]$.

When it comes to clinical manifestations, AAAs are often asymptomatic and diagnosed as an incidentaloma [10]. AAAs represent an infrequent cause of low back 


\section{EDORiUM Journals}

pain which may worsen over time in case of aneurysm expansion or impending rupture $[11,12]$.

The natural history of AAA is gradual expansion over a period of years and eventual rupture. The rate of expansion of AAA is not always linear and consistent; some patients may have stable AAA size for many years, followed by a sudden increase within a short period [13].

The pain related to a ruptured AAA is classically of sudden onset accompanied by hypotension. The mural thrombi associated with AAA may lead to distal arterial thromboembolism, eventually producing acute lower limb ischemia (the first sign in $2-5 \%$ of patients) and livedo reticularis [13].

An enlarging aneurysm may produce local mass effects due to compression of adjacent structures [13]. About 30\% of asymptomatic AAAs are found on routine consultation. Curiously, there are some reports of AAAs diagnosed on chiropractic patients $[14,15]$. During abdominal palpation, an AAA is revealed as a pulsatile, expansive mass at or above the umbilicus. The presence of a bruit may indicate aortic or visceral arterial atherosclerotic disease, or rarely an aortocaval fistula (machinery murmur). The accuracy of physical examination often depends on aneurysm size and body mass index [16]. Despite such limitations, some authors still recommend abdominal palpation and auscultation when there is suspicion of a non-mechanical cause for low back pain or history of back pain non-responsive to anti-inflammatory agents [17].

Acute AAA rupture is one of the most dramatic emergencies in medicine. Only approximately 50\% of patients with ruptured AAA reach the hospital alive; of those who reach the hospital, up to 50\% do not survive [18]. In general, endovascular AAA repair is reserved for asymptomatic aneurysms at least 5.0 to $5.5 \mathrm{~cm}$ in diameter $[18,19]$. The selection of patients should not only contemplate the estimate risk of rupture (based on the size of the aneurysm, wall thickness, intraluminal thrombus thickness, and peak wall stress) but also other parameters such as life expectancy and operative morbidity and mortality, which include coronary artery disease (leading cause of early and late mortality after AAA repair), chronic kidney disease, chronic obstructive pulmonary disease and diabetes mellitus [20].

This complexity of this vascular disorder is well demonstrated in this case report and justifies its clinical interest. In fact, CT scan imaging detected a massive pararenal AAA (which is an infrequent location, present in up to $10 \%$ of cases) with no signs of rupture in hemodynamically stable patient with a history of an insidious low back pain and without thromboembolic complications.

The patient's AAA described in this case report fits the highest cardiovascular profile due to the previous history of smoking and ischemic heart disease and it stands out for being a rare documented example of a late diagnosed AAA with a high risk of rupture submitted to successful emergency surgical treatment. This case report also highlights the importance of clinicians' awareness on the differential diagnosis of low back pain.

\section{CONCLUSION}

Aortic abdominal aneurysm represents a silent and fatal clinical condition affecting older adults with history of smoking and cardiovascular disease. It should be actively considered in the group of differential diagnosis in every male patient older than 50 years with low back pain. In case of suspicion, patient should be referred for advanced imaging investigation.

\section{REFERENCES}

1. Aggarwal S, Qamar A, Sharma V, Sharma A. Abdominal aortic aneurysm: A comprehensive review. Exp Clin Cardiol 2011 Spring;16(1):11-5.

2. Tsuchie H, Miyakoshi N, Kasukawa Y, et al. High prevalence of abdominal aortic aneurysm in patients with chronic low back pain. Tohoku J Exp Med 2013 Jun;230(2):83-6.

3. LeFevre ML; U.S. Preventive services task force. Screening for abdominal aortic aneurysm: U.S. preventive services task force recommendation statement. Ann Intern Med 2014 Aug 19;161(4):28190.

4. Ferket BS, Grootenboer N, Colkesen EB, et al. Systematic review of guidelines on abdominal aortic aneurysm screening. J Vasc Surg 2012 May;55(5):1296-304.

5. Sociedade Portuguesa de Angiologia e Cirurgia Vascular. 2016. [Available at: http://spacv.org]

6. Moll FL, Powell JT, Fraedrich G, et al. Management of abdominal aortic aneurysms clinical practice guidelines of the European society for vascular surgery. Eur J Vasc Endovasc Surg 2011 Jan;41 Suppl $1: \mathrm{S} 1-\mathrm{S} 58$.

7. Chaikof L, Brewster C, Dalman L, et al. The care of patients with an abdominal aortic aneurysm: The society for vascular surgery practice guidelines. J Vasc Surg 2009;50(4):S2-S49.

8. Lo RC, Bensley RP, Hamdan AD, et al. Gender differences in abdominal aortic aneurysm presentation, repair, and mortality in the vascular study group of new England. J Vasc Surg 2013 May;57(5):1261-8, 1268.e1-5.

9. Singh K, Bønaa KH, Jacobsen BK, Bjørk L, Solberg S. Prevalence of and risk factors for abdominal aortic aneurysms in a population-based study: The Tromsø study. Am J Epidemiol 2001 Aug 1;154(3):236-44.

10. Jarvik JG, Deyo RA. Diagnostic evaluation of low back pain with emphasis on imaging. Ann Intern Med 2002 Oct 1;137(7):586-97.

11. Van Wyngaarden JJ, Ross MD, Hando BR. Abdominal aortic aneurysm in a patient with low back pain. J Orthop Sports Phys Ther 2014 Jul;44(7):500-7.

12. Yildizgoren MT, Ogut H, Kayali A, Turhanoglu AD. Abdominal aortic aneurysm as a long time cause of low back pain and vertebral scalloping. Pain Med 2015 Aug 14. 


\section{EDORiUM Journals}

www.casereportsinternational.com

13. Lederle FA. The natural history of abdominal aortic aneurysm. Acta Chir Belg 2009 Jan-Feb;109(1):7-12.

14. Patel SN, Kettner NW. Abdominal aortic aneurysm presenting as back pain to a chiropractic clinic: A case report. J Manipulative Physiol Ther 2006 Jun;29(5):409.e1-7.

15. de Boer NJ, Knaap SF, de Zoete A. Clinical detection of abdominal aortic aneurysm in a 74-year-old man in chiropractic practice. J Chiropr Med 2010 Mar;9(1):38-41.

16. Chervu A, Clagett GP, Valentine RJ, Myers SI, Rossi PJ. Role of physical examination in detection of abdominal aortic aneurysms. Surgery 1995 Apr;117(4):454-7.

17. Mechelli F, Preboski Z, Boissonnault WG. Differential diagnosis of a patient referred to physical therapy with low back pain: Abdominal aortic aneurysm. J Orthop Sports Phys Ther 2008 Sep;38(9):551-7.

18. Golledge J, Norman PE. Current status of medical management for abdominal aortic aneurysm. Atherosclerosis $2011 \mathrm{Jul} ; 217(1): 57-63$.

19. Lederle FA, Freischlag JA, Kyriakides TC, et al. Longterm comparison of endovascular and open repair of abdominal aortic aneurysm. N Engl J Med 2012 Nov 22;367(21):1988-97.

20. Mayer D, Aeschbacher S, Pfammatter T, et al. Complete replacement of open repair for ruptured abdominal aortic aneurysms by endovascular aneurysm repair: A two-center 14-year experience. Ann Surg 2012 Nov;256(5):688-95.

\section{$* * * * * * * * *$}

\section{Author Contributions}

Jorge Vaz Lourenço - Substantial contributions to conception and design, Acquisition of data, Analysis and interpretation of data, Drafting the article, Revising it critically for important intellectual content, Final approval of the version to be published
Ricardo Marques - Substantial contributions to conception and design, Acquisition of data, Analysis and interpretation of data, Drafting the article, Revising it critically for important intellectual content, Final approval of the version to be published

Daniela Marado - Substantial contributions to conception and design, Acquisition of data, Analysis and interpretation of data, Drafting the article, Revising it critically for important intellectual content, Final approval of the version to be published

Adriano Rodrigues - Substantial contributions to conception and design, Acquisition of data, Analysis and interpretation of data, Drafting the article, Revising it critically for important intellectual content, Final approval of the version to be published

\section{Guarantor of Submission}

The corresponding author is the guarantor of submission.

\section{Source of Support}

None.

\section{Consent Statement}

Written informed consent was obtained from the patient for publication of this case report.

\section{Conflict of Interest}

Authors declare no conflict of interest.

\section{Copyright}

(C) 2018 Jorge Vaz Lourenço et al. This article is distributed under the terms of Creative Commons Attribution License which permits unrestricted use, distribution and reproduction in any medium provided the original author(s) and original publisher are properly credited. Please see the copyright policy on the journal website for more information.
Access full text article on other devices

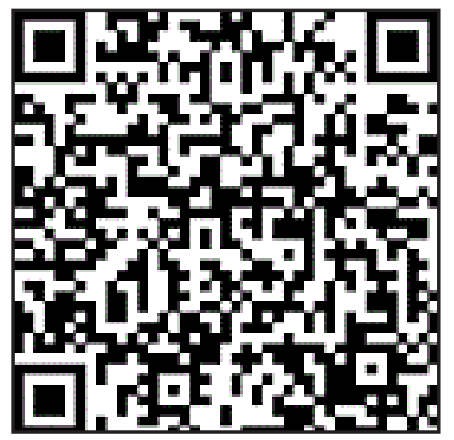

Access PDF of article on other devices

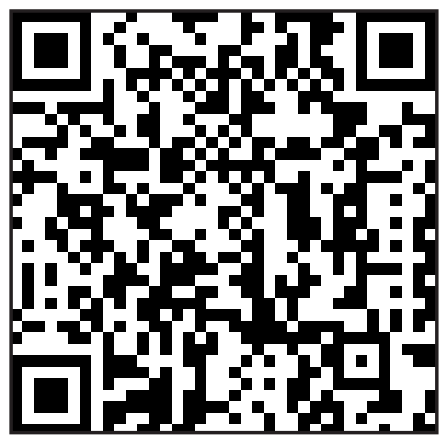

